\title{
Defining Ritualistic Driver and Passenger Behaviour to Inform In-Vehicle Experiences
}

\section{Dr. Jiayu Wu}

Intelligent Mobility Design Centre

Royal College of Art

4 Hester Road, London

SW11 4AN

jiayu.wu@rca.ac.uk

Samuel Johnson

Intelligent Mobility Design Centre

Royal College of Art

4 Hester Road, London

SW11 4AN

samuel.johnson@rca.ac.uk

\section{Katrine Hesseldahl}

Intelligent Mobility Design Centre

Royal College of Art

4 Hester Road, London

katrine.hesseldahl@network.rca.ac.uk
Daniel Quinlan

Intelligent Mobility Design Centre

Royal College of Art

4 Hester Road, London

SW11 4AN

daniel.quinlan@rca.ac.uk

Selin Zileli

Intelligent Mobility Design Centre

Royal College of Art

4 Hester Road, London

SW11 4AN

selin.zileli@network.rca.ac.uk

\section{Professor Dale Harrow}

Intelligent Mobility Design Centre

Royal College of Art

4 Hester Road, London

SW11 4AN

dale.harrow@rca.ac.uk

Permission to make digital or hard copies of all or part of this work for personal or classroom use is granted without fee provided that copies are not made or distributed for profit or commercial advantage and that copies bear this notice and the full citation on the first page. Copyrights for components of this work owned by others than the author(s) must be honored. Abstracting with credit is permitted. To copy otherwise, or republish, to post on servers or to redistribute to lists, requires prior specific permission and/or a fee.

Request permissions from Permissions@acm.org.

AutomotiveUI '18 Adjunct, September 23-25, 2018, Toronto, ON, Canada

(C) 2018 Copyright is held by the owner/author(s). Publication rights licensed to ACM.

ACM 978-1-4503-5947-4/18/09 \$15.00

https://doi.org/10.1145/3239092.3265944

\begin{abstract}
By discovering unconscious ritualistic actions in everyday driving such as preparing for the morning commute, we seek design opportunities to help people achieve critical emotional transitions such as moving from an anxious state to relief. We have gathered and analysed data from workshops and phone interviews from a variety of vehicle and public transport users to capture these key ritualistic scenarios and map their emotional transitions. Design ideation is used to generate concepts for improving the in-vehicle user experience through redesign of vehicle layout, environment and analogue and digital interfaces. We report a set of human-centred design approaches that allow us to study the details of action, objects, people, emotions and meaning for typical car users which are indispensable for designing driving experiences and are often overlooked by the car design process.
\end{abstract}

\section{Author Keywords}

Ritualistic experiences; design ideation; driver and passenger experience; user groups; scenario mapping. 


\section{Example Future Driving Scenario Rituals}

\section{Social or technology}

trend: In 2025 more people could use vehicle sharing services instead of owning their own car, a potentially even more attractive model as society ages.

Scenario: An elderly couple who live in south London are driving to a location they have never been before in Oxford. After getting into the car, they spend 10 minutes in the front seats organising the area to make themselves relaxed and comfortable for the journey, preparing for things that might happen that they are looking forward to and that they are afraid of.

Ritual achievement: The emotional transition rituals help the couple feel safe and calm. The ritualistic organisation enables them to set their expectations and prepare for the unknown journey.

\section{CCS Concepts}

Human-centered computing: Human computer interaction (HCI): HCI design and evaluation methods: user studies

\section{Introduction}

Our research is rooted from a vision of using 'emotions as affective artefacts' [7] to explore human vehicle experiences as 'artefacts are instrumental in problemsolving, decision-making and sense-making; users generate emotion as a way to minimise errors, interpret functionality, or obtain relief from the complexity of a task'. The objective of this research is to address emotions as conduit for problem solving such as relieving stress and understanding prompts and buttons, delivering sensations such as the joy of driving, and serving as a basis for decision making such as 'Should I keep my own bubble to exacerbate my loneliness while driving?' and other emotional transitions. Rituals for vehicle driving experiences emerged and attracted us as an unexplored area of automotive design with rich potential for improving driver's and passenger's emotional states. Ritual has grown from its traditional and religious roots and been extended into today's lose organised routine behaviour and habit as it has been argued that 'Many current definitions of ritual are unsatisfactory because they myopically restrict ritual experience to religious or mystical contexts' [5]. The ritualistic driving experiences we define in our research are repeated, symbolic and meaningful behaviour or performance to help emotional transition, reduce stress, refresh and reset the mind. Rituals are performed in a particular order whether ancient or modern, religious or secular. In the context of drivers or passengers, they use order to create comfort, feelings of safety and provide a sense of control. Our research aims to identify several key ritualistic driving scenarios with rich design opportunities to improve driver and passenger's experiences by managing emotional transitions.

\section{Ritual, routine and habit}

Rituals are often performed without deliberate thought to the rationale that guides them. They are 'functional through their performance, apart from their content' [2]. Ritual 'alleviates human anxiety' [3], and aims to 'get those (repeated) actions right' [4]. 'Rituals can be grand, dramatic things or personal ones. Either way, rituals help people to understand the world, cope with transitions, express strong emotions, and build their own life story' [8]. Ritual can be a 'collective sense of identity and integration among participants' [1] which forms social activities and experiences and can be very personal in everyday life. Commonly people use 'rituals' interchangeably with 'routines', but there is an important difference: unlike routines, rituals have meaning. Ritual is about moment, narrative, meaning, gestures and sequence. Habits are behaviours that people repeat and are the 'invisible architecture of daily life' [6]. In an automotive context habits include fastening a seat belt and making sure the lights are switched off after turning off the engine. Our research to date has found ritualist driver and passenger experiences are mostly focusing on settling into the car, turning on music, tidying up and cleaning the car.

\section{Methodology}

In order to identify key ritualistic driver and passenger scenarios, we conduct telephone interviews, enactment and ideation workshops, and will follow up with shadowing observations. These methods are structured to involve users in the vehicle experience design 


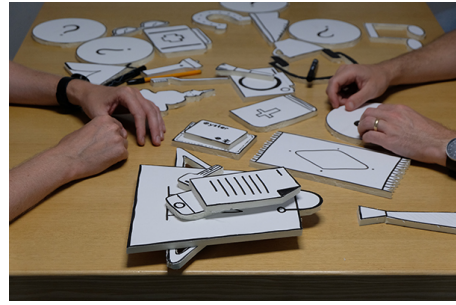

Figure 1: The cardboard props workshop participants choose and used for enacting their journey.

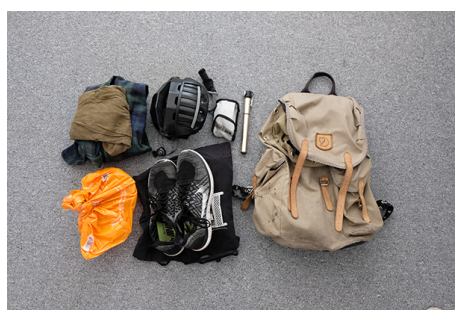

Figure 2: Workshop participants showed what objects they carry for their everyday commute.

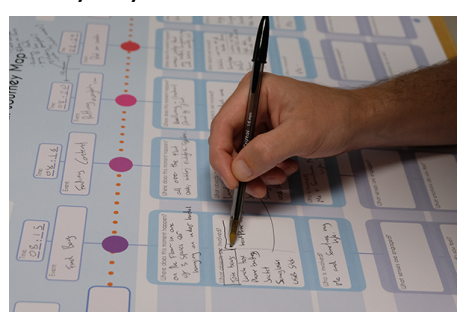

Figure 3: Journey map template participants completed with facilitators after enactment workshop role playing sessions. process with researchers and designers, recalling and reflecting on their journey details.

Telephone interviews to capture what people think about ritual/routine experiences in general and specific ritualistic experiences regarding their daily

transportation were performed with 21 drivers and public transport users ( 11 male, 10 female, 20 to 80 years old). The twenty-minute interviews covered transport scenarios including commutes, social travel, long distance travel, driving with children and car rental.

Enactment workshops looked for specific examples of ritualistic repeated behaviours that people perform before, during and after journeys. Six participants were invited in the workshops. Each workshop participant works with a research facilitator to go through a particular journey using a journey map template (figure 3) and are asked to recall a journey, name it, and identify events and times within it. The sessions include three layers of investigation to understand participant's driver or passenger experiences in depth. The first layer covers conditions of the experience such as time, action, place, objects (figure 2) and people. The second layer looks at the senses involved and emotions triggered. The third layer is for facilitator and participant to explore the meaning of the ritualistic experience such as purpose, meaning beyond function, and significance within the participant's life. Six people representing typical demographic groups from experienced driver to new driver, cyclist to walker have so far participated. They were asked to bring an object related to repeated personal or meaningful habits when they travelled to the workshop. During the workshop, they shared stories of rituals related to or beyond the object. Then they were asked to choose cardboard props (figure 1) together with the object they brought to mimic the physical movements of their journey. After the enactment session, they sat down to fill in a journey map template with the facilitator and talked about the meaning of the shared ritualistic experience and reflect on achievements.

The ideation workshops immerse designers with evidence of ritualistic transport experiences collected at the telephone interviews and enactment workshops to generate future facing scenarios for individual or socialized driver and passenger experiences taking into consideration changing technology and social trends. Follow up shadowing observations will be conducted as we identify gaps in the narrative of behaviours outlined by participants at enactment workshops. This additional detail will be used to complete the descriptions of journeys and their rituals as described to the investigators and fed through to the designers using these detailed journey maps to inform their work. Such subprojects include redesigning the layout of driver's operational environments including dashboards, central consoles and other reachable items, as our understanding of the sequence of actions as drivers prepare for driving and organise their environment in the context of their rituals improve.

\section{Initial findings}

\section{Telephone interviews}

Telephone interviews were used to understand the generalities of how people think about rituals in life and transportation and to design the enactment workshops. The findings capture a cross section of the experiences of users of public transportation and drivers of private cars, a small selection of which can be found in the sidebar on the next page. 


\section{Example telephone interview findings}

\section{Where are rituals being practiced?}

Car based rituals (38\%)

Other transport rituals (23\%) e.g. bus, tube and cycling Non-transport rituals (39\%) e.g. preparing an overnight bag, reading before sleep

\section{When do rituals happen?}

Before journeys (41\%)

During journeys (52\%)

After journeys (7\%)

\section{Popular drivers of} ritualistic experiences

Preparation and sense of accomplishment (43\%) Time to myself and emotional transition moments (33\%) Power to control (33\%)

\section{Enactment workshops}

Four example ritualistic scenarios related to driving and passenger experiences are briefly described below, with detailed information about their significance for mind resetting, mood transition, everyday life and wellbeing currently being collected to support future design work.

'Daily Me Time' refined from a 40-minute regular journey as a mother drives while listening to a podcast to pick up her daughter. She describes this is as 'me time' and 'a nice way to end the day'.

'Escapism from everyday life' refined from a 4-hour long distance trip with one hour of preparation split between the car and home for a couple and their two daughters. The preparation becomes ritualistic as the organisation of food and childcare items creates calm, satisfaction and relief. The ritual conversation between the couple is described as time 'getting back to us', 'a therapy session' and 'getting rid of daily $\mathrm{cr}^{* *}$ '.

'Building confidence for driving' refined from a 2hour stressful driving lesson. A learner driver describes coping with anxiety by arranging personal belongings, comfortable clothes, and minimising instructor conversation to aid concentration, and she always wants to look good after the stressful lessons.

'Control and safety' refined from a daily commute, the driver's ritualistic actions include switching on the satnav, dashcam, radio, head-up display and cleaning the windscreen and windows to make himself feel happy. 'I take care of the car, so it takes care of me'.

\section{Ideation workshop}

The ideation workshop ran with seven designers and researchers, generating scenarios illustrating step by step how people achieve good driving and passenger experiences, inspiring designs to fulfil their unarticulated needs. Emotional transitions, journey preparation and 'me time' were selected from the interviews and enactment workshop as directions requiring more consideration. The designers identified four future facing themes around which to sketch image-based ritualistic scenarios for informing future design opportunities for automotive design:

'Negotiation and conversation space' designs for improving how drivers and passengers dynamically interact within the front seat space.

'Mental planning and space preparation' design opportunities for settling drivers and passengers physically and mentally in the vehicle, connecting them positively with the upcoming journey.

'Playful in-vehicle actions' addressing opportunities to use vehicle spaces to encourage people to interact with inner surfaces, sounds and the outside scenery.

'Extension of driver's body' design opportunities for improving driving interfaces such as the steering wheel, pedals, gear stick, and heads up displays.

\section{Future work}

The design research team will address the four ideation workshop generated scenarios through designs which are then prototyped to test feasibility. Follow up shadowing observations will be used to better understand driver and passenger's step by step actions and difficulties. The prototype designs will be interactive objects and video demonstrations to communicate the design for ritualistic driver and passenger experiences to help the research team gain feedback from stakeholders such as automobile companies. 


\section{Acknowledgements}

This project is supported by the Hyundai Motor Group.

\section{References}

1. Eric Arnould and Linda L Price. 2000.

Authenticating acts and authoritative

performances: Questing for self and community.

The why of consumption: Contemporary

perspectives on consumer motives, goals, and

desires. Edited by S. Ratneshwar, D. G. Mick and C.

Huffman. London. Routledge, 24.

2. James H.S. Bossard and Eleanor S. Boll. 1951.

Ritual in Family Living: A contemporary Study,

Philadelphia: University of Pennsylvania Press,

1950. Social Forces, Vol. 29, Issue 3, 1951, 338.

3. Bronislaw Malinowski. 2013. Magic, science and religion and other essays. Read Books Ltd.

4. David Marshall. 2005. Food as ritual, routine or convention. Consumption, Markets and Culture, Vol. 8, No.1, March 2005, pp. 69-85.

5. Dennis W. Rook. 1985. The ritual dimension of consumer behaviour. Journal of Consumer Behaviour 12 (December): 251-64.

6. Gretchen Rubin. Better than before: Mastering the habits of our everyday lives. 2015. Two Roads An Imprint of John Murray Press. UK.

7. Frank Spillers. 2004. Emotion as a cognitive artifact and the design implications for products that are perceived as pleasurable. Experience Dynamics. USA

8. Kursat Ozenc and Margaret Hagan. 2014. Ritual Design Lab. Retrieved August 10, 2018 from https://www.ritualdesignlab.org/. 